\title{
Parallel m-Dimensional Relative Ant Colony Optimization (mDRACO) For the Costas-Array Problem
}

\author{
David Vulakh ( $\sim$ dvulakh@mit.edu ) \\ Massachusetts Institute of Technology https://orcid.org/0000-0002-7126-9547 \\ Raphael Finkel \\ University of Kentucky
}

\section{Research Article}

Keywords: CSP, Costas array, ACO, Parallelism

Posted Date: October 21st, 2021

DOl: https://doi.org/10.21203/rs.3.rs-975983/v1

License: (c) (1) This work is licensed under a Creative Commons Attribution 4.0 International License.

Read Full License

Version of Record: A version of this preprint was published at Soft Computing on March 26th, 2022. See the published version at https://doi.org/10.1007/s00500-022-06969-1. 


\title{
Parallel $m$-Dimensional Relative Ant Colony Optimization ( $m$ DRACO) for the Costas-Array Problem
}

\author{
David Vulakh* • Raphael Finkel
}

Received: date / Accepted: date

\begin{abstract}
The Costas-array problem is a combinatorial constraint-satisfaction problem (CSP) that remains unsolved for many array sizes greater than 30. In order to reduce the time required to solve large instances, we present an Ant Colony Optimization algorithm called $m$-Dimensional Relative Ant Colony Optimization (mDRACO) for combinatorial CSPs, focusing specifically on the Costas-array problem. This paper introduces the optimizations included in mDRACO, such as map-based association of pheromone with arbitrary-length component sequences and relative path storage. We assess the quality of the resulting $m$ DRACO framework on the Costas-array problem by computing the efficiency of its processor utilization and comparing its run time to that of an ACO framework without the new optimizations. $m \mathrm{DRACO}$ gives promising results; it has efficiency greater than 0.5 and reduces time-to-first-solution for the $m=16$ Costas-array problem by a factor of over 300 .
\end{abstract}

Keywords CSP · Costas array $\cdot$ ACO $\cdot$ Parallelism

\section{Introduction}

Costas arrays are square arrays of $1 \mathrm{~s}$ and 0 s such that there is exactly one element with value 1 in each row and column and no three or four distinct elements with value 1 form a

\section{Vulakh*}

Math, Science, and Technology Center, Paul Laurence Dunbar High School

E-mail: dvulakh@mit.edu

ORCiD: 0000-0002-7126-9547

R. Finkel

Computer Science Department, University of Kentucky

E-mail: raphael@cs.uky.edu

ORCiD: 0000-0003-3896-6743 (potentially degenerate) parallelogram [10]. John P. Costas first described these arrays in 1965 as representations of sequences of SONAR pulse frequencies with optimal autocorrelation properties [6]; they have since been applied in other areas, such as communication systems and cryptography [19]. Costas arrays are also objects of theoretical interest to the research community. Golomb et al. [12] describe several algebraic constructions for certain classes of Costas arrays in 1984, which remain the only known Costas-array constructions [10]. All Costas arrays of order $m \leq 29$ are known [11], but the existence of Costas arrays for many sizes $m \geq 30$ remains an open question [10].

The problem of finding Costas arrays is a constraintsatisfaction problem (CSP). CSPs are multi-variable combinatorial problems that require that each variable be assigned a value such that the assignments satisfy a set of constraints. Because there exist NP-complete CSP instances [3], no polynomial-time algorithm for the general CSP is known. In order to reduce the time to solve large CSP instances, researchers have developed parallel algorithms to pool the computational resources of multiple processors. The Costasarray problem is a common benchmark problem for assessing parallelized CSP solvers (see [1], [4], and [21] for examples).

Researchers also apply heuristic algorithms that perform stochastic or approximate searches to combinatorial problems. One family of such algorithms is the Ant Colony Optimization (ACO) metaheuristic. Modelled after the foraging patterns of ants, ACO algorithms traverse a graph of the search space, probabilistically preferring paths with components of higher learned favorability [8]. Dorigo proposed the first ACO algorithm [9] to solve constraint-optimization problems such as the Travelling Salesman Problem (TSP), but researchers have applied ACO to CSPs as well [17]. 


\subsection{Costas Arrays}

Definition 1 A constraint-satisfaction problem (CSP) [3] is an ordered triple $(V, D, C)$, where $V$ is the set of variables, $D$ is the domain 1 and $C$ is the set of constraints. Each constraint $C_{i}$ is of the form $\left(s_{i}, \rho_{i}\right)$, where $s_{i}$ describes a tuple of variables $s_{i}:\left\{1, \ldots, m_{i}\right\} \rightarrow V$ and $\rho_{i}$ is an $m_{i}$-ary relation on $D, \rho_{i} \subseteq D^{m_{i}}$.

A solution of the constraint-satisfaction problem instance $(V, D, C)$ is a function $f: V \rightarrow D$ such that for all constraints $C_{i}=\left(s_{i}, \rho_{i}\right), f \circ s_{i} \in \rho_{i}$.

In general, each variable $V_{i}$ of a CSP can have its own corresponding domain $D_{i}$. Rather than explicitly defining a domain $D_{i}$ for each variable in the definition above, we let $D=\bigcup D_{i}$ and add a unary constraint $(s, \rho)$ such that $s(1)=V_{i}$ and $\rho=D_{i}$ to each variable.

Definition 2 A Costas array [10] of size $m$ is an $m \times m$ array of 1s and 0s such that there is exactly one element with value 1 in each row and column and there are no equal displacement vectors between distinct pairs of distinct elements with value 1 (equivalently, no three or four elements with value 1 form a (potentially degenerate) parallelogram).

Costas arrays are permutation arrays, so each Costas array $A$ of size $m$ can be represented as a permutation $P$ of the integers from 1 through $m$ such that, if $A_{i j}=1$, then $P_{i}=j$. A permutation that corresponds to a Costas array in this manner is said to satisfy the Costas-array property.

The Costas-array problem is an existence problem. Whether there exist Costas arrays of size $m$ for all positive integers $m$ remains an open question [10]. The smallest $m$ for which it is unknown whether a Costas array of size $m$ exists is 32 [10].

\subsection{Ant Colony Optimization}

\subsubsection{Summary of the ACO Metaheuristic}

Ant Colony Optimization (ACO) is a class of heuristics for solving constraint-optimization problems ${ }^{2}$. In ACO algorithms, processes called ants probabilistically traverse a graph representing the search space, modifying the graph properties that determine ant behavior to increase the probability of future ants selecting paths with optimal properties. We summarize the elements of an ACO algorithm and its ant processes as described in [8].

- $C=\left\{c_{1}, \ldots, c_{n}\right\}$ is a finite set of components

\footnotetext{
${ }^{1}$ In this paper, we only consider constraint-satisfaction problems with domains of finite cardinality.

${ }^{2}$ We describe the adaptation of ACO to constraint-satisfaction problems in Section 2.1
}

- $L=\left\{l_{i j}:\left(c_{i}, c_{j}\right) \in \tilde{C}\right\}$, where $\tilde{C} \subseteq C^{2}$, is a set of connections between components

- Any finite sequence of components $s=\left(c_{i}, \ldots, c_{j}\right)$ is called a state; states need not be of a particular length or contain all components

- $S$ is the set of all possible states

- $J_{s}$ is a cost associated with the state $s$

- $\Omega$ is a set of constraints on $S$

- $P$ is a set of requirements on $S$

- $\tilde{S} \subseteq S$ is the set of all states that satisfy the constraints $\Omega ; \tilde{S}$ is then the set of feasible states

- $\psi \in \tilde{S}$ is a solution if $\psi$ satisfies all requirements $P$

- Given two states $s_{1}=\left(c_{i}, \ldots, c_{j}\right), s_{2} \in S, s_{2}$ is a neighbor of $s_{1}$ if $s_{2} \in \tilde{S}$ and there exists $c_{k}$ such that $\left(c_{i}, \ldots, c_{j}, c_{k}\right)=s_{2}$ (that is, $s_{1}$ is a prefix of $\left.s_{2}\right)$; the set $\mathcal{N}_{s}$ of states that are neighbors of $s$ is called the neighborhood of $s$

The graph $G=(C, L)$ is the construction graph. Any solution $\psi$ can be expressed as a feasible path on $G$; we seek a solution $\psi^{\prime}$ such that $J_{\psi^{\prime}}$ is minimal.

Information is associated with states $s$ (paths on $G$ ) and $i \in \mathcal{N}_{s}$ (possible next steps) in the form of pheromone values $\tau_{s i}$ and heuristic values $\eta_{s i}$. Ants, which search for the solution $\psi^{\prime}$, have the following properties:

- An ant $k$ can be assigned a start state $s_{k}$ and one or more end conditions $e_{k}$

- An ant $k$ in state $s$ can move to any component such that the resulting state $s^{\prime} \in \mathcal{N}_{s}$

- The probability $\mathcal{P}_{s i}$ that an ant $k$ in state $s$ moves to $i$ is governed by a function of $\tau_{s i}$ and $\eta_{s i}$ called the antrouting table $\mathcal{A}_{\text {si }}$

- An ant $k$ dies once it reaches an end state $e_{k}$

- If ant $k$ has started at state $s_{k}$ and after a sequence of steps has died, it has completed one iteration

ACO also executes procedures outside of the ant processes. Pheromone evaporation is the periodic decrease of all pheromone values to prevent early convergence on a suboptimal solution. Based on properties of the solutions discovered by ants, ACO also increases some pheromone values to encourage exploration of promising states. Ants indirectly communicate with each other to improve solution quality through these changes to the pheromone values.

\subsubsection{Prior Work}

ACO algorithms have been adapted to efficiently solve optimization and constraint problems in a variety of real-world and theoretical settings, including: job scheduling in mining supply chains [20]; planning selective maintenance [15]; vehicle routing [22, 24]; $k$-SAT [16]; $n$-Queens Completion [23]; and Minimum Connected Dominating Set [2]. ACO effectively solves 
optimization problems even in probabilistic settings, where the computation of the heuristic value $\eta$ can be more complicated than in simpler problems like TSP [15].

The main deficiency of ant colony algorithms in general is the tendency to prematurely converge on local minima that are globally suboptimal [7, 13]; many successful implementations of ACO achieve performance improvements by adding countermeasures against early convergence. To this end, many ACO implementations are augmented with an additional non-ACO optimization step, such as Local Search (LS) [13, 14, 20]. Performing LS on the solutions found by an ant colony can both improve convergence time by examining promising regions of the space more systematically and prevent early stagnation by sufficiently perturbing solutions that have reached a local minimum. For example, Guan et al. [14] implement an automatic updating mechanism within ACO (AU-ACO) that attempts to replace the solution constructed by each ant with a better one that differs by only one variable assignment; the resulting AU-ACO algorithm converges approximately five to ten times faster than ACO without automatic updating on binary CSP tests. Some implementations apply a more complicated LS step less frequently, such as only once the ant colony detects that it is in a stagnant state; for example, Guan et al. [13] maintain the information entropy associated with each path, initiating a crossover-based Local Search once some iteration adds little information to the system (the entropy along the best-discovered path does not change significantly). In addition to LS, Thiruvady et al. [20] consider augmenting ACO with Simulated Annealing, but find this approach ineffective. Others [2, 24] likewise apply additional mutations to discovered solutions when adapting ACO to the Minimum Connected Dominating Set and Vehicle Routing problems, respectively.

A second approach for improving ACO implementations focuses on modifying the interactions between ants by considering novel methods of manipulating the application of pheromone to the problem graph. Ye et al. 23] augment the path-construction process with a system of pheromone-based negative feedback (in addition to the standard positive feedback of ACO) to steer ants away from explored poor solutions; this ACO with negative feedback (ACON) significantly outperforms their implementation of standard ACO. Ning et al. [16] monitor the degree of similarity of solutions found in consecutive rounds and employs a pheromone-smoothing technique to escape local minima when stagnation is detected, achieving superior performance. Deng et al. [7] likewise consider a rule for diffusing pheromone applied by ants to nearby states to further encourage exploration and prevent stagnation.

\subsubsection{Our Contributions}

We present a novel ACO algorithm, $m$-Dimensional Relative Ant Colony Optimization ( $m$ DRACO). The central idea behind $m$ DRACO is to improve ACO performance by maximizing the information contained in the pheromone through which ants interact with each other; this idea contrasts with approaches that augment ACO with LS or manipulate pheromone primarily with the objective of reducing the chance of stagnation.

As discussed in Section 2.4.1 mDRACO associates pheromone with arbitrary-length subsequences of components, so the pheromone table has $m$ dimensions (where $m$ is the number of components). Typically, ACO only associates pheromone with the favorability of transitioning from one component to another, which produces a 2-dimensional table. $m$ DRACO also associates pheromone information in a relative manner, as described in Section 2.4.2 These optimizations work best when coupled with a branch-and-bound heuristic for the path-selection process, as discussed in Section 2.4.3 Our ACO implementation performs substantially better with the $m$ DRACO optimizations, reducing median run time for the Costas-array problem at $m=16$ by a factor of over 300 .

\section{Algorithm implementation}

\subsection{Adaptation of ACO to CSPs}

We apply ACO algorithms to constraint-satisfaction problems as described in [17] by treating CSPs as maximal-constraint-satisfaction problems, which are a class of constraint-optimization problems. To solve the CSP $(V, D, C)$, we let each of the components (the vertices of the graph $G$ ) represent the assignment of a single value from $D$ to a single variable in $V$. The constraints $C$ are partitioned into hard constraints $\Omega$ and soft constraints $\omega$. $\Omega$ defines the set of feasible states $\tilde{S}$ visitable by ants, and $\omega$ defines the cost of these states, so that for $s \in \widetilde{S}, J_{s}$ is the number of violations of the constraints $\omega$ in $s$.

Since ants seek the solution $\psi^{\prime}$ that has minimal cost, they seek to minimize the number of constraint violations. If $J_{\psi^{\prime}}=0$ for some $\psi^{\prime}$, then $\psi^{\prime}$ contains no violations of the constraints in $\Omega \cup \omega=C$ and therefore represents a solution to the original constraint-satisfaction problem $(V, D, C)$. In order to apply ACO to strongly constrained CSPs effectively, $C$ must be partitioned so that $\Omega$ is not so strongly constrained that a state $s \in \tilde{S}$ not representing a solution $\psi$ has $\left|\mathcal{N}_{s}\right|=0$; if the construction of feasible paths by ants is intractable, then pheromone deposition does not occur and ACO cannot exhibit its heuristic learning behavior. 


\subsection{Application to Costas arrays}

We use an ACO algorithm called the $\mathcal{M} \mathcal{A} \mathcal{X}-\mathcal{M I N}$ Ant System (MMAS) [18] as a starting point for our implementation, modelling the values $\tau_{s i}, \eta_{s i}$, and $J_{s}$ after the standard ACO TSP solution [8]. In $\mathcal{M}$ MAS, all pheromone values $\tau_{s i}$ satisfy $\tau_{\min } \leq \tau_{s i} \leq \tau_{\max }$ for some constants $\tau_{\min }$ and $\tau_{\max }$. When searching for Costas arrays of size $m$, we use $\{1, \ldots, m\}$ for the set of components, so that states (paths in $G$ ) represent sequences of integers. The sole hard constraint in $\Omega$ is that no state $s \in \tilde{S}$ contains the same component twice; the sole requirement in $P$ is that solutions $\psi$ must have length $m$. Any solution $\psi$ constructed by the ants is therefore a Hamiltonian tour of $G$ and represents a permutation of the integers from 1 through $m$.

The cost $J_{s}$ of a state $s \in \tilde{S}$ is the number of Costasarray-property violations in the permutation it represents. The heuristic value $\eta_{s i}$ is one divided by the additional cost incurred by moving to $i$ from $s$,

$$
\eta_{s i}= \begin{cases}\frac{1}{J_{i}-J_{s}} & J_{i}-J_{s}>0 \\ \eta_{\max } & J_{i}-J_{s}=0\end{cases}
$$

Here, $\eta_{\max }$ is a large constant (in our implementation, we set $\left.\eta_{\max }=2,000,000,000\right)$. When $J_{i}=J_{s}$, no new constraint violations are incurred by moving from $J_{s}$ to $J_{i}$, and any such state $J_{i}$ should be strongly preferred over one that introduces new constraint violations.

We describe the computation of $\tau_{s i}$ and $\mathcal{A}_{s i}$ in Sections 2.3 (Equation 3 and 2.4.1 (Equation 4). $\mathcal{P}_{s i}$ is the probability of selecting $i$ from $\mathcal{N}_{s}$ if each element $i$ of $\mathcal{N}_{s}$ has weight $\mathcal{A}_{s i}$,

$$
\mathcal{P}_{s i}=\frac{\mathcal{A}_{s i}}{\sum_{j \in \mathcal{N}_{s}} \mathcal{A}_{s j}}
$$

\subsection{Standard pheromone association}

Traditionally in ACO TSP Solvers, the pheromone value $\tau_{s i}$ is associated with the edge in $G$ from the last element of $s$ to the last element in $i[18]$ - that is, pheromone information represents the learned favorability of following one component with another. When the ant system deposits pheromone on a solution $\psi$, it increments $\tau_{s i}$ by $\frac{1}{J_{\psi}}$ for all $s, i$ such that the last element of $i$ follows the last element of $s$ in $\psi$ and $i \in \mathcal{N}_{s}$.

The ant-routing table $\mathcal{A}_{s i}$ for an ant in some state $s$ determining the favorability of moving to state $i$ is

$$
\mathcal{A}_{s i}= \begin{cases}0 & i \notin \mathcal{N}_{s} \\ \left(\tau_{s i}\right)^{\alpha}\left(\eta_{s i}\right)^{\beta} & i \in \mathcal{N}_{s}\end{cases}
$$

where $\alpha$ and $\beta$ are constants that determine the relative weight of pheromone and heuristics values, respectively, in the ant decision-making process.

Pheromone evaporation occurs after each ant has completed one iteration. For all states $s \in \tilde{S}, i \in \mathcal{N}_{s}$, if the pheromone value representing the learned favorability of moving to $i$ from $s$ is $\tau_{s i}$ before evaporation, it becomes $\tau_{\text {si }}^{\prime}=\max \left\{(1-\rho) \tau_{\text {si }}, \tau_{\min }\right\}$ for a constant $0 \leq \rho<1$ after.

\subsection{Optimizations in the mDRACO algorithm}

\subsubsection{Arbitrary-length pheromone association}

We attempt to increase the value of pheromone information by associating it with arbitrary-length sequences of components. To this end, we represent the set of all pheromone values deposited by ants as a map $T: \tilde{S} \rightarrow \mathbb{R}$ (implemented in our program as a multiple-reader single-writer defaultvalue hash table). When our ant system deposits pheromone on a solution $\psi$, it increments $T(s)$ by $\frac{1}{J_{\psi}}$ for all $s$ such that $s$ is a contiguous subsequence of $\psi$ of length greater than 1 . If $s=\left(c_{i}, \ldots, c_{j}, c_{k}\right)$, then the value $T(s)$ now represents the learned favorably of following an arbitrary-length sequence of components $\left(c_{i}, \ldots, c_{j}\right)$ with $c_{k}$. During evaporation, we let each map entry $T(s)$ assume the new value $\max \left\{(1-\rho) T(s), \tau_{\min }\right\}$.

We now define the pheromone information associated with moving to $i$ from $s$ as two components: $\tau_{s i}$ (pheromone value) and $\lambda_{s i}$ (trail length value). If $\tau_{0}$ is the default map value ${ }^{3}$ and $j$ is the longest non-empty suffix of $i$ for which $T(j) \neq \tau_{0}$, then $\tau_{s i}=T(j)$ and $\lambda_{s i}$ is the length of $j$. If there is no such suffix, then $\tau_{s i}=\tau_{0}$ and $\lambda_{s i}=1$.

We define the ant-routing table $\mathcal{A}_{s i}$ for an ant in state $s$ determining the favorability of moving to state $i$ :

$$
\mathcal{A}_{s i}= \begin{cases}0 & i \notin \mathcal{N}_{s} \\ \left(\tau_{s i}\right)^{\alpha}\left(\eta_{s i}\right)^{\beta}\left(\lambda_{s i}\right)^{\gamma} & i \in \mathcal{N}_{s}\end{cases}
$$

where $\alpha, \beta$, and $\gamma$ are constants that determine the relative weight of pheromone, heuristic, and trail-length values, respectively, in the ant decision-making process.

We expect that longer pheromone trails carry more meaningful information, which is why we choose always to use the value in $T$ associated with the longest suffix of $i$ when determining $\tau_{s i}$. However, the value mapped by $T$ to a long pheromone trail $j$ is less than or equal to the value mapped by $T$ to all suffixes of $j$, since a greater number

\footnotetext{
${ }^{3}$ In accordance with the standard $\mathcal{M M A S}$ algorithm, we initialize the default map value $\tau_{0}$ to $\tau_{\max }$ and let this value evaporate after each iteration as though it were a map entry. At some point early in the search, $\tau_{0}$ assumes a permanent value of $\tau_{\min }$. To keep map memory consumption from growing without bound over the course of the program's execution, we remove entries that, as a result of pheromone evaporation, map to $\tau_{\min }$, which should be positive.
} 
of paths constructed by ants share shorter subsequences than longer ones. To account for this imbalance, we add the $\left(\lambda_{s i}\right)^{\gamma}$ factor in the ant-routing table entry.

Note: In the original manuscript, sections 2.4 .2 and 2.4.3 were presented in the opposite order.

\subsubsection{Relative path storage}

Because we expect two sequences for which the difference between corresponding elements is constant (e.g. $(3,1,2,5)$ and $(4,2,3,6)$ ) to have related favorability in constructing Costas arrays, we further modify the process of pheromone association by using a canonical version of each state. If $s$ is a sequence of integers with first element $s_{0}$, let the canonical sequence of $s z^{s}$ be the sequence with the same length as $s$ such that if the $i$ th element of $s$ is $s_{i}$, the $i$ th element of $z^{s}$ is $z_{i}^{s}=s_{i}-s_{0}$. Now, whenever looking up or modifying the value that $T$ maps to a sequence $s$, we use $T\left(z^{s}\right)$. The result is that an ant considering the learned favorability of $(3,1,2,5)$ pools information collected about all $s$ such that $z^{s}=z^{(3,1,2,5)}=(0,-2,-1,2)$, including $(4,2,3,6)$. We do not expect this heuristic to improve $m$ DRACO run time for combinatorial problems in general, since the numeric values assigned to domain elements are arbitrary for problems like the TSP. However, we do expect this optimization to generalize to other problems with costs based on relative positions of domain values, such as the N-Queens Problem.

\subsubsection{Quality threshold}

Some ants may, during the path construction process, arrive at a state $s$ such that $J_{s}$ is high and $s$ is still far from being a solution (that is, $s$ is short). Ants in such a position waste run time and pheromone map queries in finishing the construction of $s$, since the cost of any solution $\psi$ with $s$ as a prefix is necessarily at least $J_{s}$. To reduce resources wasted in this way, we apply a branch-and-bound heuristic by setting a quality threshold $\vartheta$ for the ants such that, if $\psi^{\prime}$ is the lowest-cost solution found by the ant system and an ant is located at state $s$ with $J_{s}>J_{\psi^{\prime}}+\vartheta$, the ant dies without finishing the path $s$ and creates a new path when respawned.

\section{Assessment}

We implement the ant system described in the $\mathrm{C}++$ language using the pthread and latomic implementations of STL headers <thread> and <atomic $>$. We conduct our assessments by running this implementation on a Xeon Phi 7210 processor (Knight's Landing) with 64 cores supporting 256 threads, provided by the University of Kentucky Center for Computational Sciences. The machine runs CentOS 8. Throughout the experimentation

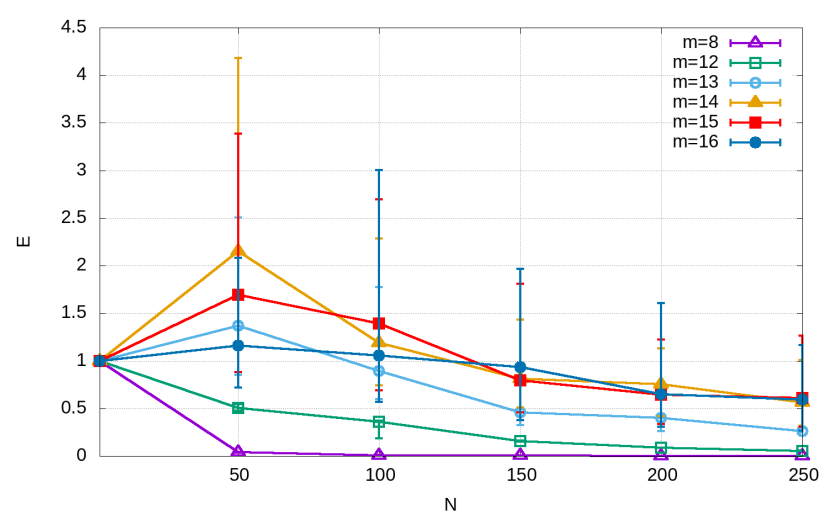

Fig. 1 Median $E$ measured as a ratio of times to first solution (over 100 runs) for each number of workers $N$ and problem size $m$. Error bars at the first and third quartiles

process, we saturate the logical processors of the machine, employing approximately 250 threads at a time.

\subsection{Efficiency}

A standard metric of parallel-program performance is efficiency, a measure of the amount of parallel computational resources effectively applied to the solution; as the number of workers increases, the efficiency tends to decrease. The definition below describes specifically how we computed the values shown in Figure 1 .

Definition 3 (Efficiency) If a parallel ant system with a single worker solves a problem with median time to first solution $T_{0}$, and an execution of a parallel ant system with $N$ workers finds a (possibly different) first solution to that problem in time $T$, then the speedup $S$ and efficiency $E$ of the $N$-worker system for that execution are

$$
S=\frac{T_{0}}{T} \quad E=\frac{S}{N}=\frac{T_{0}}{N \cdot T}
$$

The definitions we give differ slightly from the traditional ones in sources such as [5] on several points. First, since the mDRACO program is stochastic and the time to first solution varies across runs, we define speedup and efficiency to be properties of a single execution of the ant system rather than ones of the program as a whole. Second, while traditional definitions compare the run time of a parallel program running on $N$ processors to that of its serialization, we choose to use a parallel ant system with one worker (and therefore two total processes) to determine $T_{0}$, because serializing our parallel ant system would require a change in the methods of communication between workers and the queen. 
The efficiency results depicted in Figure 1 show $E>0.5$ for $m \geq 14$. $E$ decreases monotonically as $N$ increases. The values of $E>1$ are due to the stochastic nature of the ant system; some runs of the algorithm are lucky, leading to a quick discovery of a solution. The consistent value of $E \approx$ 0.6 for $m \geq 14$ and $N \geq 200$ implies that the efficiency is likely to remain greater than 0.5 for larger problem sizes $m$ and worker counts $N$. In our measure of efficiency, $N$ is the number of workers utilized by the program. Even the $N=1$ case has two processors available to it, so we expect optimal efficiency to be closer to 0.5 than to 1 .

\subsection{Optimized performance}

To assess the quality of the three optimizations: arbitrarylength pheromone association, relative path storage, and the quality threshold $\vartheta$, we compare the median time-to-firstsolution over 100 runs of our implementation of $m$ DRACO with various combinations of these optimizations. In all cases, the parameters $\alpha, \beta, \gamma, \tau_{\min }$, and $\tau_{\max }$ are held constant. All constants are set to experimentally-determined optimal values.

Figure 2 shows the median time-to-first-solution in seconds for problem sizes $12 \leq m \leq 19$, and Figure 3 visualizes these data over $12 \leq m \leq 16$. Weaker combinations of settings take too long to complete for data collection to be reasonable; for example, out of a small sample of 2,0,NR runs on the size-17 Costas-array problem, only one finished within 50,000 seconds. For this reason, some table entries are left empty.

Though each of the optimizations is fairly weak on its own, the experimental results show that they are very powerful when applied together, and their usefulness appears to increase rapidly with problem size. Run-time differences are generally negligible for $m<15$, and the slightly poorer performance of some of the optimized ACO variants is likely a result of overhead that does not pay off on easier problem instances, where solutions can often be found without much pheromone guidance. For $m \geq 15$, the differences in run times between different settings of combinations become pronounced. At $m=15, m$ DRACO (the rightmost column of Figure 2 finds solutions approximately 50 times faster than our standard ACO implementation; at $m=16$, $m$ DRACO is approximately 300 times faster. We do not have a reliable estimate of the median time-to-first solution of standard ACO for $m=17$ for the reasons described above; however, if we assume that it is at least 50,000s, $m$ DRACO is at least 3,000 times faster at $m=17$.

Interestingly, arbitrary-length pheromone association on its own (the second column of Figure 2 performs significantly worse than our implementation of standard ACO. To ensure that the good performance of mDRACO is not simply the result of the combination of the other two optimiza-

\begin{tabular}{c|cccccc}
$m$ & $2,0, \mathrm{NR}$ & $0,0, \mathrm{NR}$ & $2,20, \mathrm{NR}$ & $2,0, \mathrm{R}$ & $2,20, \mathrm{R}$ & $0,20, \mathrm{R}$ \\
\hline 12 & 0.11 & 0.11 & 0.11 & 0.11 & 0.11 & 0.11 \\
13 & 0.11 & 0.11 & 0.11 & 0.11 & 0.11 & 0.11 \\
14 & 0.13 & 0.15 & 0.13 & 0.20 & 0.12 & 0.21 \\
15 & 26.75 & 168.60 & 0.52 & 15.55 & 0.66 & 0.57 \\
16 & 910.36 & 3628.63 & 789.08 & 472.17 & 8.45 & 2.92 \\
17 & - & - & - & - & 132.98 & 17.75 \\
18 & - & - & - & - & 9147.29 & 95.99 \\
19 & - & - & - & - & - & 798.75
\end{tabular}

Fig. 2 Median time-to-first solution in seconds (over 100 runs) for our implementation of $m$ DRACO with various combinations of optimizations enabled. The columns are labelled by triples $\lambda, \vartheta, r . \lambda=2$ indicates that pheromone is only associated with the transition from one state to the next (the standard ACO behavior), while $\lambda=0$ indicates that pheromone is associated with arbitrary-length sequences of components ( $m$ DRACO behavior). $\vartheta=0$ indicates that the branch-andbound heuristic is not used (standard ACO behavior), while $\vartheta=20$ indicates a quality threshold of 20 ( $m$ DRACO behavior). $r=\mathrm{NR}$ indicates that the pheromone association is not relative (standard ACO behavior), while $r=\mathrm{R}$ indicates that it is relative ( $m$ DRACO behavior). The rightmost column contains the results for the full $m \mathrm{DRACO}$ algorithm.

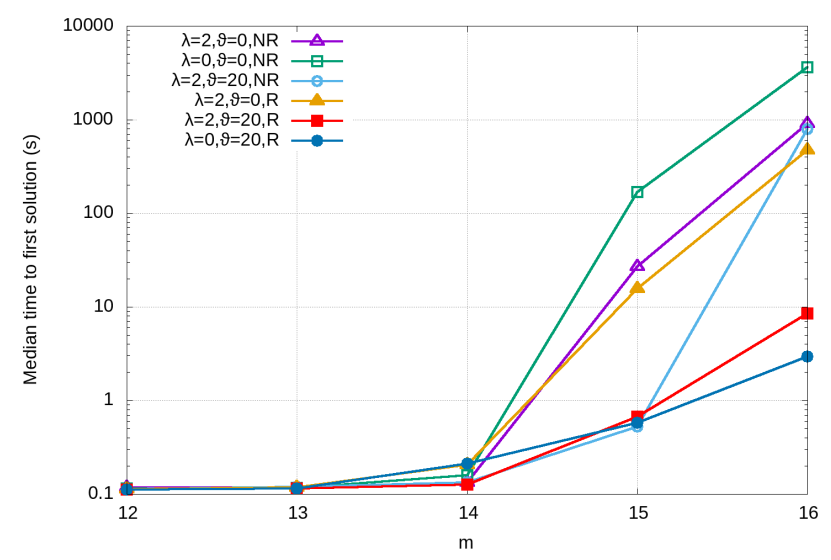

Fig. 3 Median time-to-first solution for our implementation of $m$ DRACO over $12 \leq m \leq 16$, as in Figure 2 The $y$-axis is logarithmic.

tions, we also conduct experiments using only relative path storage and a $\vartheta=20$ quality threshold (the fifth column from the left in Figure 21. This combination of optimizations is $\sim 2.9$ times slower than $m$ DRACO for $m=16$, $\sim 7.5$ times slower for $m=17$, and $\sim 95$ times slower for $m=18$. We speculate that arbitrary-length pheromone association hurts ACO performance on its own but significantly improves run times when coupled with the other two optimizations because it makes the process of performing map lookups and insertions much more expensive, as every subsequence of the path generated by an ant is inserted to the map when a path is constructed, and every suffix must be checked when computing $\tau$. This overhead overwhelms any benefits that can be gained from the additional information carried by pheromone when much effort is expended on the fruitless paths pruned early by a well-chosen $\vartheta$. 


\section{Conclusion}

We present a novel implementation of the ACO metaheuristic, $m$ DRACO, that finds solutions to Costas-array problem for sizes $m \leq 19$ while maintaining high efficiency $(E>0.5)$ even at large worker counts $(N=250)$ and reduces median time-to-first solution by several orders of magnitude for relatively large problem sizes. We have applied $m$ DRACO to the unsolved $m=32$ size of the Costasarray problem - at the time of writing, the ant system has used over 10,000 days of CPU time and discovered a permutation of the integers from 1 to 32 with 17 Costas-arrayproperty violations (shown in Appendix A.

The program as written is generally applicable to any finite-domain CSP. We expect that the optimizations described in 2.4 generalize to some other CSPs and constraint-optimization problems. Specifically, we expect that arbitrary-length pheromone association (Section 2.4.1) improves the performance of ACO algorithms on combinatorial problems that can be solved with the typical TSP pheromone association scheme, such as the TSP itself, graph-coloring problems, and the N-Queens Problem. Relative path storage (Section 2.4.2) likely generalizes to problems in which constraints or costs relate to relative positions of domain values, such as the N-Queens Problem. Since the branch-and-bound quality threshold $\vartheta$ (Section 2.4.3) can be used to reduce wasted resources for any problem, we expect that it generalizes to all ACO solvers.

Further investigation is necessary to determine exactly which classes of combinatorial problems benefit from the three optimizations introduced by $m \mathrm{DRACO}$, and to what extent. We also have not yet studied how these optimizations behave when used simultaneously with other known ACO variants, such as when they are coupled with Local Search heuristics, pheromone diffusion [7], or negative pheromone feedback [23]. We have observed that the three optimizations introduced in this paper interact with each other in unexpected ways, so studying their interaction with other modifications may be an especially interesting direction of future research.

The $\mathrm{C}++$ source for our $m \mathrm{DRACO}$ program is available at https: / / github.com/dvulakh/mDRACO

\section{A Arrays discovered}

After over 10,000 CPU-days on a Knight's Landing computer, our $m$ DRACO program has found these approximate solutions to the Costas-array problem with $m=32$.

19 violations

8192520171013141626232283122464311130211529231185972227 18 violations

1412253013161726233520719109312182432827461292128221511 9152028178222912253230161811213731191423127244261325106
1416234269101918111732311527211231282481323029725520622 17 violations

2731231814315226296432252281024716175121120281302191913

\section{Declarations and Compliance with Ethical Standards}

Funding: This study was funded by the Kentucky Young Researchers Program.

David Vulakh declares that he has no conflict of interest.

Raphael Finkel declares that he has no conflict of interest.

Ethical approval: This article does not contain any studies with human participants or animals performed by any of the authors.

\section{Data Availability}

The software analyzed in this paper is available at https://github.com/dvulakh/mDRACO

The datasets generated and analyzed during the current study are available from the corresponding author on reasonable request.

\section{References}

1. Arbelaez, A., Codognet, P.: A GPU implementation of parallel constraint-based local search. In: 2014 22nd Euromicro International Conference on Parallel, Distributed, and Network-Based Processing, pp. 648-655. IEEE (2014)

2. Bouamama, S., Blum, C., Fages, J.G.: An algorithm based on ant colony optimization for the minimum connected dominating set problem. Applied Soft Computing 80, 672-686 (2019)

3. Bulatov, A.A., Krokhin, A.A., Jeavons, P.: Constraint satisfaction problems and finite algebras. In: International Colloquium on Automata, Languages, and Programming, pp. 272-282. Springer (2000)

4. Caniou, Y., Codognet, P., Richoux, F., Diaz, D., Abreu, S.: Largescale parallelism for constraint-based local search: the costas array case study. Constraints 20(1), 30-56 (2015)

5. Cormen, T.H., Leiserson, C.E., Rivest, R.L., Stein, C.: Introduction to algorithms. MIT press (2009)

6. Costas, J.P.: A study of a class of detection waveforms having nearly ideal range-Doppler ambiguity properties. Proceedings of the IEEE 72(8), 996-1009 (1984)

7. Deng, W., Xu, J., Zhao, H.: An improved ant colony optimization algorithm based on hybrid strategies for scheduling problem. IEEE access 7, 20281-20292 (2019)

8. Dorigo, M., Di Caro, G.: Ant colony optimization: a new metaheuristic. In: Proceedings of the 1999 congress on evolutionary computation-CEC99 (Cat. No. 99TH8406), vol. 2, pp. 1470-1477. IEEE (1999)

9. Dorigo, M., Maniezzo, V., Colorni, A.: Ant system: optimization by a colony of cooperating agents. IEEE Transactions on Systems, Man, and Cybernetics, Part B (Cybernetics) 26(1), 29-41 (1996)

10. Drakakis, K.: Open problems in Costas arrays. arXiv preprint arXiv:1102.5727 (2011)

11. Drakakis, K., Iorio, F., Rickard, S., Walsh, J.: Results of the enumeration of Costas arrays of order 29. Adv. in Math. of Comm. 5(3), 547-553 (2011)

12. Golomb, S.W., Taylor, H.: Constructions and properties of Costas arrays. Proceedings of the IEEE 72(9), 1143-1163 (1984)

13. Guan, B., Zhao, Y., Li, Y.: An Ant Colony Optimization Based on Information Entropy for Constraint Satisfaction Problems. Entropy 21(8), 766 (2019) 
14. Guan, B., Zhao, Y., Li, Y.: An improved ant colony optimization with an automatic updating mechanism for constraint satisfaction problems. Expert Systems with Applications 164, 114021 (2021)

15. Liu, Y., Chen, Y., Jiang, T.: On sequence planning for selective maintenance of multi-state systems under stochastic maintenance durations. European Journal of Operational Research 268(1), 113127 (2018)

16. Ning, J., Zhang, Q., Zhang, C., Zhang, B.: A best-path-updating information-guided ant colony optimization algorithm. Information Sciences 433, 142-162 (2018)

17. Roli, A., Blum, C., Dorigo, M.: ACO for maximal constraint satisfaction problems. In: Proceedings of MIC, vol. 1, pp. 187-191 (2001)

18. Stützle, T., Hoos, H.H.: MAX-MIN ant system. Future generation computer systems 16(8), 889-914 (2000)

19. Taylor, K., Rickard, S., Drakakis, K.: Costas arrays: survey, standardization, and matlab toolbox. ACM Transactions on Mathematical Software (TOMS) 37(4), 1-31 (2011)

20. Thiruvady, D., Ernst, A.T., Singh, G.: Parallel ant colony optimization for resource constrained job scheduling. Annals of Operations Research 242, 355-372 (2016)

21. Truchet, C., Richoux, F., Codognet, P.: Prediction of parallel speed-ups for las vegas algorithms. In: 2013 42nd International Conference on Parallel Processing, pp. 160-169. IEEE (2013)

22. Yan, F.: Autonomous vehicle routing problem solution based on artificial potential field with parallel ant colony optimization (ACO) algorithm. Pattern Recognition Letters 116, 195-199 (2018)

23. Ye, K., Zhang, C., Ning, J., Liu, X.: Ant-colony algorithm with a strengthened negative-feedback mechanism for constraintsatisfaction problems. Information Sciences 406, 29-41 (2017)

24. Zhang, H., Zhang, Q., Ma, L., Zhang, Z., Liu, Y.: A hybrid ant colony optimization algorithm for a multi-objective vehicle routing problem with flexible time windows. Information Sciences 490, 166-190 (2019) 


\section{Supplementary Files}

This is a list of supplementary files associated with this preprint. Click to download.

- ref.bib

- spmpsci.bst

- svglov3.clo

- svjour3.cls 